gruppenanalysen. Dabei verzichtet sie weitestgehend auf „Kochrezepte“ zugunsten einer differenzierten Darstellung der Vor- und Nachteile verschiedener Techniken. Da in Umfrageexperimenten aufgrund der Randomisierung auf große Mengen an Kontrollvariablen verzichtet werden kann, empfiehlt sie darüber hinaus mehr Wert auf die Reliabilität der Messinstrumente zu legen. Daneben diskutiert Mutz auch die ethischen Probleme, die mit der Täuschung von Versuchspersonen verbunden sind. Kapitel 7 widmet sich der statistischen Analyse und Interpretation von Umfrageexperimenten. Behandelt werden Randomisierungs-Checks, die Gewichtung experimenteller Daten und die Verwendung von Kovariaten. Die externe Validität als Hauptvorteil von Umfrageexperimenten steht im Mittelpunkt des dritten Teils. Als besonders gelungen kann Kapitel 8 gelten. Im Gegensatz zu einer weit verbreiteten Praxis plädiert $M u t z$ dafür, die externe Validität einer Untersuchung nicht alleine am Forschungsdesign oder dem Setting (Labor, Feld, Umfrage) festzumachen, sondern als kumulativen Forschungsprozess zu begreifen. Anschließend diskutiert sie die Generalisierbarkeit von Umfrageexperimenten, wobei sie nicht nur die üblichen Dimensionen wie Untersuchungssituation und Teilnehmer berücksichtigt, sondern auch, inwiefern die eingesetzten Treatments und Operationalisierungen verallgemeinert werden können. Das letzte Kapitel fasst die wesentlichen Erkenntnisse noch einmal kurz zusammen und schließt mit einer knappen wissenschaftstheoretischen Bewertung von Umfrageexperimenten, die ruhig hätte länger ausfallen können. In der Gesamtschau bietet Diana Mutz eine kompetente und leicht verständliche Einführung in die Thematik. Wie bereits erwähnt, wird dabei aber ein gewisses Basiswissen in experimentellen Methoden und Umfrageforschung vorausgesetzt. Insofern ersetzt das Buch nicht das Studium entsprechender Lehrbücher, sondern eignet sich eher als ergänzende Lektüre. Der erste Teil des Buches ist vor allem eine Sammlung an interessanten und kreativen Ideen für Experimente, wobei die Autorin leider häufig darauf verzichtet, methodisch in die Tiefe zu gehen. Wenn man selbst an der konkreten Anwendung einzelner Designs interessiert ist, sollte man hier eher die Originaluntersuchungen $\mathrm{zu}$ Rate ziehen. Die Stärken des Buches liegen eindeutig im zweiten und dritten Teil, die mit einer Vielzahl praktischer Tipps aufwarten können und Aspekte diskutieren, die man in dieser Dichte und Aktualität sonst nicht findet. Kritisch anzumerken bleibt, dass diese Kapitel durch einen Abschnitt ergänzt werden könnten, der sich mit den Auswirkungen sinkender Teilnahmebereitschaft an Umfragen auf die externe Validität beschäftigt. Alles in allem ist das Buch aber ein wertvolles Nachschlagewerk für alle, die Umfrageexperimente planen, durchführen oder auswerten möchten.

Alexander Glantz

\section{INTERNATIONALE POLITIK}

\section{Dür, Andreas. Protection for Exporters. Power and Discrimination in Transat- lantic Trade Relations, 1930-2010. Ithaca, NY. Cornell University Press 2010. 246 Seiten. $34,99 €$.}

Warum wurde der transatlantische Handel seit den 1930er Jahren liberalisiert? Warum haben sich die USA und 
die europäischen Länder, insbesondere die EU, für mehr Handelsliberalisierung als andere Länder entschieden? Allgemeiner formuliert: Warum nimmt Handelsliberalisierung verschiedene Formen an, und warum war Nicht-Diskriminierung zwischen ausländischen Vertragspartnern von 1930 bis 1960 wichtiger als heute?

Andreas Dür argumentiert in seinem Buch Protection for Exporters. Power and Discrimination in Transatlantic Trade Relations, dass sich Handelsliberalisierung mit dem Einfluss von exportorientierten Interessengruppen erklären lässt. Exportorientierte Interessengruppen mobilisieren sich immer dann, wenn sie durch präferentielle Handelsabkommen mit Marktzugangsverlusten konfrontiert werden, und sie üben Druck auf ihre Regierungen aus, um Zugang zu diesen Märkten zu bewahren. Dies wiederum führt zu einer Veränderung der Präferenzen von Regierungen, die nun versuchen, die Interessen von Exporteuren zu schützen. Im zweiten Schritt seiner Argumentationskette hebt Dür hervor, dass Regierungen der ausgeschlossenen Länder zum Schutz der Interessen ihrer exportorientierten Unternehmen insgesamt vier Strategien verfolgen können: Sie können erstens mit Vergeltungsmaßnahmen drohen; zweitens ein alternatives Abkommen unterzeichnen; drittens ein Abkommen mit Ländern, die Handel in einer nicht-diskriminierenden Weise liberalisieren, unterzeichnen; und viertens dem vorhandenen bilateralen Abkommen beitreten. Dabei geht der Autor davon aus, dass die Auswahl einer diesen vier Optionen vom Vulnerabilitätsgrad eines ausgeschlossenen Landes abhängt. Der dritte Schritt des Arguments gibt dann eine Antwort auf die Frage, warum die Un- terzeichner von präferentiellen Handelsabkommen externe Handelsliberalisierung akzeptieren und auf die Forderungen der ausgeschlossenen Länder eingehen. Dür begründet dies damit, dass die Unterzeichnung eines präferentiellen Handelsabkommens die Verhandlungsmacht der Unterzeichnerstaaten erhöht, wodurch sie zusätzliche Zugeständnisse von den ausgeschlossenen Ländern bekommen können. Dies bedeutet, dass Handelsdiskriminierung externe Effekte hat und zu einer Reaktion der Nichtunterzeichnerstaaten führen kann. Im Mittelpunkt der „Schutz-für-die-Exporteure-These“ stehen drei Fragen: Warum kommt es zur Mobilisierung der Exporteure gegen Verluste, aber nicht für potentielle Gewinne? Mit welchen Strategien reagieren die Regierungen der ausgeschlossenen Länder darauf? Unter welchen Bedingungen kann sich ein Nichtunterzeichnerstaat erfolgreich für die Interessen seines exportorientierten Sektors einsetzen?

Um Antworten auf diese Fragen zu geben, stellt der Autor fünf Hypothesen auf, die sich mit folgenden Determinanten beschäftigen: Mobilisierungsgrad von Exporteuren nach der Unterzeichnung eines präferentiellen Handelsabkommens, Einfluss von exportorientierten Interessengruppen, Auswahl der Regierungsstrategie, Verhandlungsmacht einer Regierung und die Bereitschaft der Unterzeichnerländer eines präferentiellen Handelsabkommens, sich auf Verhandlungen mit einem ausgeschlossenen Land einzulassen. Diese Hypothesen werden im empirischen Teil der Studie überprüft. Dür untersucht sechs Fallstudien, die einen Zeitraum von 80 Jahren umfassen: Die Entstehung des britisch-imperialen Präferenzsystem und die Reaktion der 
USA, 1932-1947; Pattsituationen in transatlantischen Handelsverhandlungen, 1948-1957; Europäische Wirtschaftsgemeinschaft und transatlantische Beziehungen, 1958-1963; Auswirkung der ersten EG-Erweiterung auf die US-Handelspolitik; Einheitliche Europäische Akte (EEA) und transatlantische Handelsbeziehungen in den 80er Jahren; Wettbewerb zwischen der EU und den USA, 1995-2010.

Die Fallstudien vermitteln eine sehr detaillierte Darstellung der US-Handelspolitik seit den 1930er Jahren bis heute, insbesondere in Bezug auf die Rolle der exportorientierten Interessengruppen. Darin liegt auch eine der Schwächen der Studien: Durch den starken Fokus auf die US-Handelspolitik erfährt der Leser weniger über die EG/ EU-Handelspolitik. Diese wird auf die Präferenzen von zwei Staaten (Frankreich und Deutschland) reduziert. Des Weiteren ist bei der fünften Fallstudie, wie der Autor selbst zugibt, die Erklärungskraft der „Schutz-für-die-Exporteure-These" gering. Wenn präferentielle Handelsabkommen die Verhandlungsmacht der Unterzeichnerstaaten erhöhen, warum entscheiden sie sich für multilaterale Handelsliberalisierung, wie dies in der Uruguay-Runde des GATT und der Doha-Runde der WTO geschah? Auch die sechste Fallstudie liefert keine überzeugende Evidenz für die "Schutz-für-die-Exporteure-These". Der Autor argumentiert zwar, dass die Zunahme des Aushandelns von präferentiellen Handelsabkommen, vor allem seitens der EU, zu einer Mobilisierung der US-Exporteure führte, die dann im Jahr 2002 in ein Handelsvollmacht-Gesetz (trade promotion authority bill) mündete. Dieses ermächtigte den US-Präsidenten, Handelsabkommen auszuhandeln, welche beschleunigt vom US-Kongress beraten werden (fast track). Die Studie kann zwar insgesamt gut erklären, inwiefern das Interesse der USA an bilateralen und regionalen Handelsabkommen in den späten 1980er Jahren (US-Kanada und US-APEC) eine Reaktion auf die Vertiefung europäischer Integration, insbesondere auf die EEA, war. Aber die EU und die USA reagieren zunehmend weniger aufeinander, sondern eher auf Drittstaaten, insbesondere auf Schwellenländer. Dadurch wird der Fokus auf transatlantische Handelsbeziehungen etwas problematisch. Dies sind nur drei unwesentliche Kritikpunkte, welche die Qualität dieser Studie in keiner Weise beeinträchtigen. Insgesamt ist es dem Autor hervorragend gelungen, Theorie mit empirischer Evidenz zu verknüpfen. Andreas Dür hat ein exzellentes Buch über die transatlantischen Handelsbeziehungen von 1930 bis 2010 geschrieben. Durch seine Langzeitperspektive und den Vergleich der EU- und US-Handelspolitiken mit speziellem Fokus auf den Einfluss von Interessengruppen, hat der Autor ein Referenzwerk der Internationalen Politischen Ökonomie geschrieben, das von allen gelesen werden sollte, die sich mit Handels- und Kooperationsfragen befassen.

Eugénia da Conceição-Heldt

Harnisch, Sebastian, Cornelia Frank und Hanns W. Maull (Hrsg.). Role Theory in International Relations. Approaches and Analyses. Abingdon. Routledge 2011. 322 Seiten. $95 €$.

Rollentheoretische Ansätze haben in der Theorie der Internationalen Beziehungen lange Zeit keine prominente Rolle gespielt. Dies ist - wie die Her- 